\title{
Strafanzeige bei Gewalttätigkeit von psychisch Kranken während der stationären Behandlung - Pro \& Kontra
}

\author{
Criminal Prosecution of Assaultive Psychiatric Patients - Pro \& Contra
}

Pro

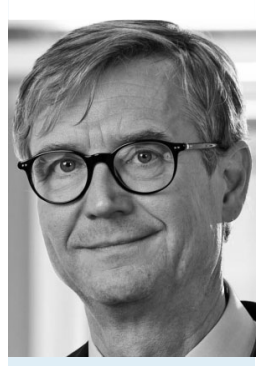

Harald Dreßing

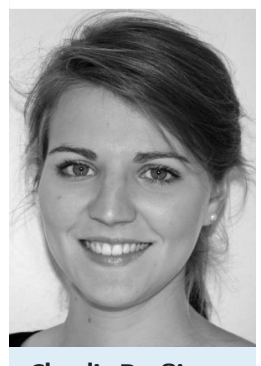

Claudia Dreßing
Die jährliche Rate von gewalttätigen Angriffen gegenüber Ärzten, Therapeuten und Pflegepersonal in psychiatrischen Kliniken ist mit 62 Vorkommnissen pro 1000 Beschäftigten im Jahr hoch [1]. Bis in die 1970er-Jahre war es fester Bestandteil des professionellen Selbstverständnisses, dass solche Angriffe einen obligaten Bestandteil des therapeutischen Geschehens darstellen und als solche von den Behandlern auch zu dulden sind.

Während ähnlich gewalttätige Handlungen außerhalb des klinischen Settings regelmäßig zu Strafanzeigen führten, wäre es für die Therapeuten undenkbar gewesen, dass sie ihre Patienten für dieselben Handlungen anzeigen würden, wenn diese sich während eines stationären Aufenthaltes ereigneten. Im Jahre 1978 wurde in der psychiatrischen Literatur erstmals von einer Strafanzeige gegen einen gewalttätigen Patienten berichtet [2]. Seitdem wird die Thematik kontrovers diskutiert [3]. Der Zusammenhang zwischen einzelnen psychiatrischen Krankheiten (z.B. schizophrene Psychosen, Substanzabhängigkeit) und einem erhöhten Risiko für gewalttätiges Verhalten ist mittlerweile gut belegt [4]. Die derzeitige psychiatrische Versorgungspraxis mit einer immer kürzeren stationären Verweildauer und einer zunehmenden Betonung der Patientenautonomie führt dazu, dass Therapeuten in der psychiatrischen Klinik zunehmend auch mit gewalttätigem Verhalten von Patienten konfrontiert sind und eine Strafanzeige als eine mögliche und adäquate Reaktion diskutieren. Grundsätzlich gibt es aus Sicht der Autoren keinen Grund, von einer Strafanzeige abzusehen, nur weil sich das gewalttätige Verhalten im Krankenhaus ereignet hat. Bei dieser Pro-Position ist zu beachten, dass die Autoren keinesfalls von einem Automatismus zur Strafanzeige ausgehen, sondern ein solches Vorgehen für eher seltene und gut begründete Ausnahmefälle in Betracht ziehen, nachdem die relevanten ethischen, medizinischen und rechtlichen Aspekte gründlich erörtert wurden.

Eine Strafanzeige ist grundsätzlich dann in Betracht zu ziehen, wenn ein stationär behandelter Patient sich gegenüber dem Personal oder gegenüber Mitpatienten gewalttätig verhalten hat und eine professionelle Risikoeinschätzung zu dem Ergebnis kommt, dass von ihm eine weitere Gefahr ausgeht. Das Hauptmotiv für eine Strafanzeige in einem solchen Fall gründet nicht in einem Vergeltungs- oder Bestrafungsbestreben, sondern basiert auf dem Gedanken der Spezial- und Generalprävention, künftige Übergriffe durch die Stärkung des Legalverhaltens der Patienten sowie des Vertrauens in die Funktionsfähigkeit der Rechtsordnung abzuwehren. Man mag einwenden, dass es mit der Rolle der Therapeuten ethisch unvereinbar sei, einen Patienten anzuzeigen. Allerdings ist die psychiatrische Therapie anders als andere medizinische Behandlungen häufig mit einem doppelten Mandat versehen. Einerseits besteht der Auftrag, dem psychisch kranken Patienten zu helfen, andererseits sind immer auch Aspekte einer krankheitsbedingten Fremdgefährdung $\mathrm{zu}$ beachten, für deren $\mathrm{Ab}$ wehr mit den jeweiligen Landesunterbringungsgesetzen auch ein rechtlicher Handlungsrahmen und eine Handlungsverantwortlichkeit zur Verfügung steht. Das Allgemeine Strafrecht bietet und gebietet gerade vor dem Hintergrund der Gleichheit vor dem Gesetz auch für den besonderen Bereich der stationären Behandlung den Rückgriff auf die allgemeingültigen Grundsätze, um die Grenze zwischen strafbarem und straffreiem Verhal- ten zu ermitteln. Es kann nicht davon ausgegangen werden, dass stationär behandelte Patienten für gewalttätiges Verhalten automatisch unzurechnungsfähig sind. Hier kommt es auf eine sorgfältige Einzelfallprüfung an und selbst bei psychotisch erkrankten Patienten können antisoziale Einstellungen und Motive im Vordergrund stehen.

In begründeten Einzelfällen kann es über den Weg einer Strafanzeige auch zu einer Verlegung eines Patienten in eine forensische Klinik kommen, wenn dessen Schuldfähigkeit krankheitsbedingt erheblich beeinträchtigt ist. Möglich ist in einem solchen Fall auch, dass eine Unterbringung im Maßregelvollzug angeordnet, aber zur Bewährung ausgesetzt wird, was eine kontinuierliche Behandlung in der Allgemeinpsychiatrie unter Umständen erst ermöglicht. So gesehen kann eine Strafanzeige durchaus auch im Sinne des Patienten sein, da auf diesem Weg unter Umständen eine erfolgreiche Behandlung eingeleitet werden kann und der Patient durch eine Therapie von weiteren krankheitsbedingten Straftaten abgehalten wird. Es geht dabei nicht darum, unliebsame Patienten in die Forensische Psychiatrie abzuschieben. Selbstverständlich sollen krankheitsbedingt fremdaggressive Patienten vorrangig in der Allgemeinpsychiatrie behandelt werden. In begründeten Einzelfällen ist eine Strafanzeige aber nicht nur rechtlich geboten und ethisch vertretbar, sondern auch im wohlverstandenen Interesse des betroffenen Patienten, der Mitpatienten und des Behandlungsteams.

Ein weiterer Einwand könnte darin gesehen werden, dass durch eine Strafanzeige die Schweigepflicht gebrochen wird. Allerdings gibt es eine Vielzahl anderer Konstellationen, in denen die Schweigepflicht gegen andere Rechte abgewogen werden muss, insbesondere wenn es um den Schutz Dritter geht. Für eine Strafanzeige ist es auch nicht notwendig, Einzelheiten aus der Therapie zu berichten, es ist völlig ausreichend, den Vorfall und die Person 
zu benennen, sodass bereits der objektive Tatbestand eines möglichen Verstoßes gegen $§ 203$ StGB ausscheidet. Sofern man als Behandler Kenntnis von geplanten schweren Straftaten erhält (z.B. Mord, Totschlag) besteht im Rahmen des $§ 138$ StGB sogar eine rechtliche Pflicht zur Durchbrechung der Schweigepflicht. Es gibt bisher für die dargestellte Problematik kein standardisiertes und allgemein akzeptiertes Vorgehen und es mangelt auch an empirischer Forschung zu dem Thema [5]. Dies führt auch zu einer gewissen Rechtsunsicherheit und im Zweifel macht man dann lieber gar nichts. Man will seine therapeutische Rolle und den Ruf der Klinik nicht beschädigen und möglichen rechtlichen Konsequenzen aus dem Weg gehen. Auf der Basis des derzeitigen Kenntnisstandes [6] stellen die folgenden Kriterien eine sinnvolle Entscheidungsgrundlage dar:

1. Strafanzeigen gegen psychisch kranke, stationär behandelte Patienten sind seltenen Ausnahmefällen vorbehalten.

2. Es sollte eindeutig definierte Prozeduren geben, in die der Klinik- und Verwaltungsdirektor der Klinik einbezogen sind.

3. Ein Kollege mit forensisch-psychiatrischer Expertise sollte zur Risikoeinschätzung konsultiert werden.

Eine Strafanzeige kommt aus Sicht der Autoren insbesondere dann in Betracht, wenn
- ein Patient ein erheblich gewalttätiges oder wiederholt gewalttätiges Verhalten gezeigt hat

- eine Gewalteskalation zu prognostizieren ist

- es zu sexuellen Übergriffen gekommen ist

- klinische Interventionsmöglichkeiten (z.B. Medikation, Isolierung etc.) ausgeschöpft sind

- davon auszugehen ist, dass das öffentliche Interesse an der Strafverfolgung von der Staatsanwaltschaft bejaht wird. Den rechtlichen Rahmen formt $§ 158$ I StPO: Demnach kann Jedermann eine Straftat zur Anzeige bringen, unabhängig davon, ob er selbst Verletzter ist [7]. Dies berechtigt sowohl das Krankenhauspersonal, aber vor allem auch die juristische Person des Krankenhauses selbst, den strafrechtlich relevanten Sachverhalt zur Anzeige zu bringen. Insbesondere letzteres ist empfehlenswert, da hierdurch sowohl das Behandlungsverhältnis geschützt als auch die persönliche Belastung des Krankenhauspersonals verringert werden kann.

Davon abzugrenzen ist der Strafantrag, den lediglich so genannte Antragsdelikte i.S. der $\S \S 77-77$ d StGB erfordern. Relevant ist besonders $\S 230$ StGB, der den Antragsdeliktscharakter von Körperverletzungen normiert. Hier muss der Verletzte selbst einen Strafantrag stellen, sonst sind die Prozessvoraussetzungen eines Strafverfahrens nicht erfüllt und die Tat wird nicht verfolgt.

\section{Literatur}

1 Anderson A, West SG. Violence Against Mental Health Profesionals: When the Treater Becomes the Victim. Innov Clin Neurosci 2011; 8: 34-39

2 Schwarz CJ, Greenfield GP. Charging a patient with assault of a nurse on a psychiatric unit. Canadian Psychiatric Association Journal 1978; 23: 197-200

3 Appelbaum KL, Appelbaum PS. A Model Hospital Policy on Prosecuting Patients for Presunptively Criminal Acts. Hospital and Community Psychiatry 1991; 42: 1233-1237

4 Fazel S, Langström N, Hjern A et al. Schizophrenia, Substance Abuse, and Violent Crime. JAMA 2009; 301: 2016-2032

5 van Leeuwen ME, Harte JM. Violence against care workers in psychiatry: is prosecution justified? Int J Law Psychiatry 2011; 34: 317-323

6 Ho J, Ralston DC, McCullough LB et al. When should psychiatrists seek criminal prosecution of assaultive psychiatric inpatients? Psychiatr Serv 2008; 60: 1113-1117

7 Hannich R. Karlsruher Kommentar zur Strafprozessordnung. 6. Aufl. 2008

Korrespondenzadresse

Prof. Dr. Harald Dreßing

Zentralinstitut für Seelische Gesundheit

J 5

68159 Mannheim

harald.dressing@zi-mannheim.de

Bibliografie

DOI http://dx.doi.org/

10.1055/s-0032-1327312

Psychiat Prax 2013; 40: 11-12

(c) Georg Thieme Verlag KG

Stuttgart · New York

ISSN 0303-4259 